\title{
A first attempt at ordinal projective measurement ${ }^{\star}$
}

\author{
Jacob Denolf \\ Department of Data Analysis, Ghent University, \\ H. Dunantlaan 1, 9000 Ghent, Belgium \\ jacob.denolf@ugent.be
}

\begin{abstract}
To our knowledge, all applications of the quantum framework in social sciences are used to model measurements done on a discrete nominal scale. However, especially in cognition, experiments often produce data on an ordinal scale, which implies some internal structure between the possible outcomes. Since there are no ordinal scales in physics, orthodox projection-valued measurement (PVM) lacks the tools and methods to deal with these ordinal scales. Here, we sketch out an attempt to incorporate the ordinal structure of outcomes into the subspaces representing these outcomes. This will also allow us to reduce the dimensionality of the resulting Hilbert spaces, as these often become too high in more complex quantum-like models. To do so, we loosen restrictions placed upon the PVM (and even POVM) framework. We discuss the two major consequences of this generalization: scaling and the loss of repeatability. We also present two applications of this approach, one in game theory and one concerning Likert scales.
\end{abstract}

Keywords: Quantum-like measurement, Ordinal scales, Likert scales

\section{Introduction}

With the emerging success of applying the quantum probabilistic toolbox in social sciences, there is also an increasing focus on its limitations. In physics, the construction of the needed model is relatively straightforward. However, in quantum cognition, the quite rigid recipe sometimes shows its limits both mathematically and interpretationally ([9]). So, it shouldn't come as a surprise that more recent work tries to expand the reach of these tools by looking at possibilities beyond the standard projective measurement (PVM) principles. The best known generalization beyond PVM is the use of Positive Operator Valued Measurement (POVMs) ([2]), but alternative, sometimes even more general, approaches also arise (e.g. [11], [1]). These ventures are mostly theoretical in nature, with applications using experimental data being rather sparse. None

\footnotetext{
* The author would like to thank Kirsty Kitto, James Yearsley, Ariane LambertMogiliansky and especially Ismael Martínez-Martínez for the engaging discussions and comments.
} 
of these approaches, however, deals with the problem of representing outcomes with an internal structure.

In this paper we present an idea which also goes beyond orthodox quantumlike techniques. This new technique was originally formulated for a specific setting in [10] and further developed and tested in the recently submitted [7]. In these two papers, a model is constructed which deals with the relationship of a participant's beliefs and preferences in a game theoretic setting, taken from [3]. During this process, problems concerning a too high dimension of a Hilbert space arose, which where solved by drawing inspiration from a rotational solution presented in [15] and (ab)using the ordered structure of the possible outcomes. To do so, we opted to loosen certain restrictions which lead to alternative types of projectors. While the solution to these problems served an ad hoc purpose, the question if this new technique could be applied in different settings presented itself.

Here we argue that this generalization of $\mathrm{P}(\mathrm{O}) \mathrm{VM}$ can be used to model any situation where different outcomes of a measurement have an internal ordinal structure. After defining this generalization, we discuss two consequences of using this new structure and present two possible applications of this approach: the game theoretic one mentioned before and Likert scales in general.

\section{Revisiting the Clinton/Gore example}

We take a new look at the quantum-like model concerning public opinion on Bill Clinton and $\mathrm{Al}$ Gore. This is one of the go-to introductory examples in quantum cognition, see for example [4]. In a Gallup poll, conducted September 6-7, 1997, participants were asked 2 separate questions: if they think Clinton is trustworthy and if they think Gore is trustworthy. When the Clinton question is posed first, $53 \%$ of the participants consider him to be trustworthy and $73 \%$ consider Gore to be trustworthy. However, when the question order is reversed, $67 \%$ think Gore is trustworthy and $59 \%$ think Clinton is trustworthy. This change in attitude indicates an order effect, which suggests a quantum-like approach by considering the Clinton and Gore questions to be incompatible. In the resulting quantumlike model each question is represented by an orthogonal 2 dimensional basis, with each vector representing the relevant 'yes' or 'no' answer and by defining a 2-dimensional Hilbert space containing both bases. The resulting model has a good statistical fit, with only two parameters (one coordinate of the state vector, as the second coordinate is fixed due to the normalization restriction, and one angle between the two bases) to be estimated.

We now identify two properties of this experimental paradigm, which become problematic when we leave this relative simple example for more complex ones. First, the number of possible outcomes is low. Both questions only allow 2 possible replies, while trustworthiness of presidential candidates could be considered far more complex. This gives the resulting Hilbert space a manageable two dimensions. Note that as all measurements are considered incompatible, no tensoring is required, which would increase dimensionality exponentially. Sec- 
ond, there is no structure in the outcomes. The yes and no outcomes are on a discrete nominal scale, with no implicit relationship between them.

Let's make the situation a bit more complex. First, suppose we want to add some more nuance to the questions and allow for more replies: very trustworthy/quite trustworthy/somewhat trustworthy/neutral/somewhat untrustworthy/quite untrustworthy/very untrustworthy. These outcomes clearly have an internal structure, as they are ordered. This extension makes the resulting Hilbert space 7dimensional. Second, suppose that, for whatever research reasons, a third similar measurement is performed, which also allows for a similar set of 7 outcomes, that does not produce order effects. Even though the situation is not extreme from an experimental point of view, the Hilbert space needed to model this situation would be 49-dimensional. This would increase the amount of parameters needed to fit the state vectors and subspaces dramatically, resulting in an inoperable model. Next to this unwieldy dimensionality, this approach lacks the tools to incorporate the ordinal structure of the outcomes. Since, to our knowledge, no ordinal scales ${ }^{1}$ are present in quantum mechanics, where would these tools come from? However, in contrast, ordinal scales are widespread in psychology, with their own distinct theory, framework and statistics.

In what follows, we propose a first attempt at modeling ordinal outcomes, within the quantum-like approach. This attempt also reduces the problematic dimensionality that arises when measurements with more than two outcomes are performed and tensoring is needed, when constructing the relevant bases.

\section{Defining the new ordinal projectors}

Paraphrasing Kirsty Kitto in her QI15 talk, see [2], a quantum(-like) measurement $\boldsymbol{M}$, with its set of possible outcomes $\left\{\boldsymbol{M}_{i}\right\}$, is represented by a set of subspaces $\left\{\mathcal{M}_{i}\right\}$, where $\mathcal{M}_{i}$ represents outcome $\boldsymbol{M}_{i}$. These subspaces $\mathcal{M}_{i}$ each define a projector $P_{i}$, which projects any vector $|S\rangle$ on the relevant subspace $\mathcal{M}_{i}$. The state of a system (e.g. a participant in a psychological experiment) is represented by a normalized state vector $|\psi\rangle$. Now, the mathematical rules are quite straightforward:

(i) The probability of obtaining outcome $\boldsymbol{M}_{i}$ is $\left\langle\psi\left|P_{i}\right| \psi\right\rangle$ or, intuitively, the closer the state vector is to the relevant subspace, the higher the probability of obtaining that outcome.

(ii) After obtaining outcome $\boldsymbol{M}_{i}$, the state after measurement becomes $\frac{P_{i}|\psi\rangle}{\sqrt{\left\langle\psi\left|P_{i}\right| \psi\right\rangle}}$ or, intuitively, when obtaining an outcome, the state vector becomes a normalized vector in the relevant subspace.

As is widely known, the orthodox quantum measurement paradigm (Projectionvalued measurement or PVM) demands that all subspaces associated with one measurement are orthogonal and, perhaps trivially, that these subspaces span the entire Hilbert space. This ensures that probabilities sum to one and that when

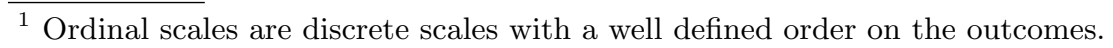


a measurement is performed twice, without any manipulation in between both measurements, the same outcome is obtained twice. We call this last property repeatability. Perhaps less widely known, when we weaken the demand that all subspaces associated with one measurement are orthogonal but still ensure that all probabilities sum to one by demanding that all relevant projector matrices sum to the identity matrix:

$$
\sum_{i} P_{i}=I
$$

we obtain a more general class of measurements which we call Positive OperatorValued Measurement (POVMs). Note that POVMs do not adhere to repeatability. This first generalization gives us freedom to incorporate structure in the outcomes, while reducing the dimensionality. However, this solution is still more restrictive then one might think at first, as the $\sum_{i} P_{i}=I$ demand is still quite strong. More concrete, when a set of outcome vectors is defined, typically an extra outcome vector has to be introduced to ensure all projectors sum to the identity matrix. Take, as an example, a simple two dimensional case. When two non-orthogonal vectors $\left|M_{1}\right\rangle=(1,0)$ and $\left|M_{2}\right\rangle=(\cos \theta, \sin \theta)$, with projectors

$$
P_{1}=\left(\begin{array}{ll}
1 & 0 \\
0 & 0
\end{array}\right) \text { and } P_{2}=\left(\begin{array}{cc}
\cos ^{2} \theta & \cos \theta \sin \theta \\
\cos \theta \sin \theta & \sin ^{2} \theta
\end{array}\right)
$$

are needed to model an experimental situation, their projectors sum to:

$$
P_{1}+P_{2}=\left(\begin{array}{cc}
1+\cos ^{2} \theta & \cos \theta \sin \theta \\
\cos \theta \sin \theta & \sin ^{2} \theta
\end{array}\right)
$$

Having the diagonal elements equal to one can easily be achieved by appropriate scaling. However, to have the off-diagonal elements equal to zero, a third outcome vector $\left|M_{3}\right\rangle=( \pm \cos \theta, \mp \sin \theta)$ or $\left|M_{3}\right\rangle=( \pm \sin \theta, \mp \cos \theta)$ must be introduced, even when there is no third possible experimental outcome!

To solve this, we propose to omit the demand that all projectors sum to the identity matrix, effectively losing almost all structure, but use this freedom to add new structure which reflects our ordinal scale, while still adhering to our basic quantum-like rules (i) and (ii). The necessity of generalizing measurement beyond POVMs is not a new idea, as remarked in [9] and discussed in chapter 8 of $[14]$.

As we only have two mathematical entities at hand (a state vector $|\psi\rangle$ and a set of subspaces $\left\{\mathcal{M}_{i}\right\}$ representing outcomes), this structure has to be incorporated in these two. On the one hand, as the state vector is supposed to represent the particular state of the system, the type of scale of the measurement should not impact this state vector. On the other hand, as the set of subspaces is representing the outcomes, any structure between these outcomes, should be reflected in a structure between the subspaces. This is why we allow subspaces associated with outcomes of the same measurement to be non-orthogonal to each other. Now we can define the notion of a subspace $\mathcal{M}_{i}$ being closer to a subspace 
$\mathcal{M}_{j}$ then to a subspace $\mathcal{M}_{k}$, when $\widehat{\mathcal{M}}_{i} \mathcal{M}_{j}$, the angle ${ }^{2}$ between $\mathcal{M}_{i}$ and $\mathcal{M}_{j}$, is smaller then $\widehat{\mathcal{M}}_{i} \mathcal{M}_{k}$ the angle between $\mathcal{M}_{i}$ and $\mathcal{M}_{k}$. This gives us a natural way of representing an ordinal scale with outcomes $\boldsymbol{M}_{i}$ (admitting to a well defined order $\prec$ ) by demanding that :

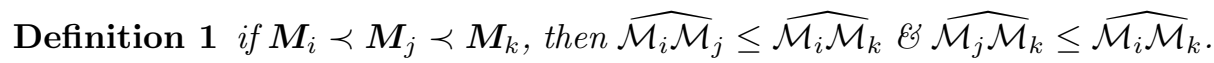

Note that the maximum angle between two subspaces is $\pi / 2$, so orthogonal subspaces are considered to be the farthest away possible from each other.

The exact value of these angles is an empirical question, which we discuss later. When all relevant subspaces are orthogonal, each subspace adds its own dimension to the total dimension of the encompassing Hilbert space, which is the reason of the exploding dimensionality in the introductory example. As the need for orthogonality is now omitted, the resulting dimensionality can be greatly reduced as compared to the traditional PVM approach. This makes the dimension of the final Hilbert space also an empirical question and/or a deliberate choice, taking into account, e.g., the number of data points or certain demands for elegance or simplicity of the resulting model. The concepts for calculating probabilities (i) and post-measurements states (ii) remain identical to the ones used with PVMs and POVMs. Note that as all considered $P_{i}$ are projectors, they are still Hermitian positive semi-definite, so $\left\langle\psi\left|P_{i}\right| \psi\right\rangle$ is positive and real. Because the state vector still gets projected on the subspace representing the obtained outcome, this approach keeps the quantum-like nature. As a result, all concepts (order effects, contextuality, entanglement...) used in quantum cognition are still a part of this approach because the regular PVM structure is now a specific case of our more general framework.

\section{Consequences}

Our loosening of restrictions used when defining $\mathrm{P}(\mathrm{O}) \mathrm{VMs}$ has significant consequences. Here, we discuss the major two.

\subsection{Sum of probabilities}

As we do not require restriction (1) to hold, it is possible that the sum of the possibilities across all possible outcomes exceeds 1 . While this seems problematic at first, two solutions naturally present themselves. First, a scaling factor can be introduced. This is the solution used in [7]. Keeping the notations defined as in the previous section, for all $|\psi\rangle$ define $C_{M}$ as:

$$
C_{M}=\sum_{j}\left\langle\psi\left|P_{j}\right| \psi\right\rangle
$$

\footnotetext{
$\overline{{ }^{2} \text { The angle }} \widehat{\mathcal{M}_{i} \mathcal{M}_{j}}$ between two subspaces $\mathcal{M}_{i}$ and $\mathcal{M}_{j}$ is classically defined as $\min \left(\widehat{V_{i} V_{j}}\right)$, with $V_{i} \in \mathcal{M}_{i}$ and $V_{j} \in \mathcal{M}_{j}$.
} 
This allows us to scale appropriately. Now, we redefine the probability of obtaining outcome $\boldsymbol{M}_{i}$ as

$$
\begin{aligned}
P^{\prime}\left(\boldsymbol{M}_{i}\right) & =\frac{P\left(\boldsymbol{M}_{i}\right)}{C_{M}} \\
& =\frac{\left\langle\psi\left|P_{i}\right| \psi\right\rangle}{\sum_{j}\left\langle\psi\left|P_{j}\right| \psi\right\rangle} .
\end{aligned}
$$

This gives us

$$
\begin{aligned}
\sum_{i} P^{\prime}\left(\boldsymbol{M}_{i}\right) & =\frac{\sum_{i}\left\langle\psi\left|P_{i}\right| \psi\right\rangle}{\sum_{j}\left\langle\psi\left|P_{j}\right| \psi\right\rangle} \\
& =1 .
\end{aligned}
$$

While this approach lacks mathematical elegance, it effectively makes the probabilities sum to one.

A second, more elegant, solution is inspired by classical logistic regression. In logistic regression, a function $f\left(x_{1} \ldots x_{n}\right)$ is derived, where, given a number of predictors $x_{1} \ldots x_{n}$, the outcome of a binary variable $(A$ or $\neg A)$ is estimated. The natural way of predicting a binary outcome would be to estimate the probability of obtaining $A$. However, as there is no way to ensure that the image of the derived function $f\left(x_{1} \ldots x_{n}\right)$ is a subset of $[0,1]$ (the same problem as with our non-orthogonal subspaces) the odds $\frac{P(A)}{P(\neg A)}$ are modeled, instead of the probability $P(A)$. Since odds only have the restriction that they are positive, this approach can also be successfully introduced here:

$$
\begin{aligned}
\operatorname{ODDS}\left(\boldsymbol{M}_{i}\right) & =\frac{P\left(\boldsymbol{M}_{i}\right)}{P\left(\neg \boldsymbol{M}_{i}\right)} \\
& =\frac{\left\langle\psi\left|P_{i}\right| \psi\right\rangle}{\left\langle\psi\left|I-P_{i}\right| \psi\right\rangle} .
\end{aligned}
$$

Using odds does not introduce any new factors, making it more elegant mathematically. One can easily calculate standard probabilities from these odds since the scaling factor needed beforehand would disappear throughout the calculations. However, odds might be more difficult to interpret. To our knowledge, there are no quantum-like models where these odds are used. It can be easily shown by calculating the odds with the newly defined $P^{\prime}\left(\boldsymbol{M}_{i}\right)$ that both solutions are identical from a modeling point of view.

\subsection{Loss of repeatability}

As a consequence of allowing non-orthogonal subspaces to represent outcomes of the same measurement, we lose repeatability: when a measurement is performed twice, without any manipulation between both measurements, two different outcomes can be obtained. While repeatability seems a necessity at first, multiple 
instances where it is not required (or is even considered too strict) can be found in, among other fields, cognition. The best known approach lacking repeatability is the use of POVMs, which we defined in section 3. For an in-depth discussion of the use of POVMs in cognition and the relationship to repeatability, we refer to [2] and [9]. More on the application of POVMs in physics can be found in [13]. Summarizing, models not adhering to repeatability are not only feasible, but also sometimes required within quantum cognition.

What could this loss of repeatability mean within our Clinton/Gore example and ordinal scales in general? When we go back to our 7 outcome ordinal scale 'very trustworthy/quite trustworthy/somewhat trustworthy/neutral/somewhat untrustworthy/quite untrustworthy/very untrustworthy', we claim that some of these outcomes should not exclude each other. To justify this, we introduce the notion of unsharp measurement. This idea is already successfully implemented in [7]. We claim that when participants are forced to pick one of these outcomes, their reply does not mean a complete dismissal of another option as these opinions are not completely distinguishable (see also the discussion of 'distinguishing quantum states in 2.2.4 of [14]). When, e.g., a participant replies that he thinks Gore is somewhat trustworthy, the participant does not necessarily disagree with the notion that Gore is quite trustworthy. The more probable it is that two options do not preclude each other, the closer their respective vector spaces should be. While the example might be too simple and underestimating the cognitive abilities of the participants, there is always a tipping point where outcomes do become psychologically indistinguishable. To construct an extreme example, suppose that the trustworthiness question allows for an ordinal scale ranging from 1 (untrustworthy) to 1000 (trustworthy). There is no participant that could successfully fathom the difference between, e.g., replying 503 and replying 504. The internal structure we incorporated, ensures that if repeatability is violated in such cases, the possible outcomes of the repeated questions are neatly scattered around the original answer, as the closer two subspaces are, the more likely it is that the outcomes they represent are obtained after each other. The upper limit case of this is the original outcome, which has the highest probability of being obtained again. The lower limit case of this are outcome vectors orthogonal to the vector representing the original outcome. They can not be obtained in the repeated measurement. As such, the class of measurements where repeatability does occur, is a subclass of the one we propose, by having all relevant outcome vectors orthogonal.

Note that this idea of unsharp measurement can be empirically tested. To do so, simply confront the participant with a different option than the given reply and ask if the participant could agree with it. These ideas allow the model to be constructed in an empirical way: test or argue which outcomes are mutually exclusive and represent these by orthogonal subspaces (this also determines the dimension of the resulting Hilbert space). Observe which outcomes are not excluded and define their subspaces accordingly. We illustrate this type of reasoning in the second example of the next section. Moreover, this approach allows for statistical testing of certain cognitive hypotheses concerning cognitive abili- 
ties and/or ordinal scaling by checking if allowing these 'close' subspaces results in (more) satisfying statistical fits of experimental data.

\section{Applications}

\subsection{QP\&B model}

A first example where these new types of projectors on non-orthogonal subspaces have already been constructed and successfully applied was presented in [10] and later expanded upon in [7] (including a successful statistical test against experimental data). The incentive of constructing them in this paradigm, lay in the emergence of a problematic dimensionality when following the traditional quantum-like course. A thorough overview of the game theoretic experiment and the resulting model falls outside the scope of this paper. The relevant part in this discussion concerns the part of the experiment where participants are asked to estimate how much of their 9 possible opponents in a game choose to cooperate (as opposed to defect) in a certain step of the experiment. As this measurement allows 10 different outcomes $(0 \ldots 9$ opponents believed to be cooperating), it naturally leads to a 10 dimensional Hilbert space. Due to the relationship between other measurements performed in this experiment, two of these Hilbert spaces need to be tensored, resulting in a (problematic) 100dimensional Hilbert space. Internal structure is clearly present in the replies, as, e.g., replying ' 6 ' is closer to replying ' 7 ' then it is to replying ' 8 '. Combined with an argument that the ' 0 ' and ' 9 ' replies should exclude each other, which forces the subspaces representing ' 0 ' and ' 9 ' to be orthogonal, the resulting subspaces are defined in a 2-dimensional Hilbert space, with reply ' $i$ ' being represented by vector $\left|B_{i}\right\rangle$, as can be seen in figure 1. Defining the participant's beliefs as projections of the state vector in the same plane doesn't differ much from rotating the state vector by using a Hamiltonian, as is done in a similar prisoner dilemma setting in [5]. However, we opted to still derive probabilities from our projectors, as opposed to just using the rotation for representing a time evolution, as in [5]. This approach reduced the problematic dimensionality, with the final dimension equal to 4, while still retaining the advantages quantum-like models provide (such as modeling order effects) and yielded a very good statistical fit.

It is worth mentioning briefly that the above situation can also be modeled using a POVM structure, with an extra outcome, as mentioned when discussing POVMs in section 3. This approach is taken from [17], where it is described in detail. To do so we keep the definition of the 10 outcome vectors $\left|B_{i}\right\rangle$ as beforehand and define an ad hoc new outcome vector $\left|B_{f}\right\rangle$, representing that 'the measurement has failed', similar to the vector $\left|M_{3}\right\rangle$ in section 3. This $\left|B_{f}\right\rangle$ ensures that all projector matrices sum to the identity matrix. The probabilities in this case are redefined as the probabilities of obtaining a certain outcome on the condition that the measurement didn't fail. When 'the measurement failed' outcome would be (theoretically) obtained, the measurement is supposed to redone immediately. The resulting probabilities are identical to the probabilities 


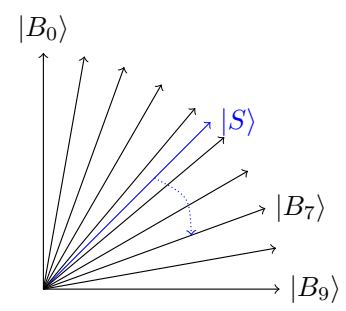

Fig. 1. The outcome 'i' is represented by $\left|B_{i}\right\rangle$. The participant is represented by state vector $|S\rangle$. Here, the participant replies that he thinks 7 opponents have cooperated, projecting/rotating the state vector onto $\left|B_{7}\right\rangle$.

obtained by using our new non-POVM ordinal structure. Note that in this experimental setting the measurement never fails, therefor the POVM structure does not represent the experiment in a clean way. This poses a question to the modeler: to stay within the bounds of POVM structures, at the cost of not naturally representing the experiment or to stray beyond POVM structure, but achieving a straightforward representation of the experiment.

\section{$5.2 \quad$ Likert scales}

A second natural candidate for this treatment is the modeling of Likert scales (for an overview on Likert scales, see [16]). Likert scales are used in polling of opinions and consist of multiple Likert items. A Likert item consists of a statement, which the participant evaluates on a given scale. This scale should be symmetric (a neutral option and and equal number of positive and negative options) and balanced (the perceived distance between following options is equal). The format of a typical five level Likert item looks like

strongly disagree (1) - disagree (2) - neutral (3) - agree (4) - strongly agree (5),

which is clearly on an ordinal scale. These Likert scales are widely used in Psychology in general and in opinion polling surveys in particular. The use of quantum-like techniques when dealing with these kind of surveys is already established, as, e.g., they are prone to order effects ([12]). Some work has already been done to use quantum-like techniques when dealing with Likert scales ([6]). However, this approach suffers from the two problems flagged before. First, the dimension of the used Hilbert spaces gets high very quickly and second, the implicit ordinal structure of the outcomes is represented in the state vector, which should only represent the participant, and not in the outcome vectors. Our view opens up new possibilities to tackle these Likert scales. We construct one as an example, but keep in mind that this particular form has not been tested against any experimental data. We only wish to take some first steps to showcase the flexibility of our approach. When looking at the (1)-(5) scale presented above, we argue that 'strongly disagree (1)', 'neutral (3)' and 'strongly agree (5)' should 
exclude each other, as we consider them in our example as non-nuanced, very clear opinions . As such, they are represented by orthogonal vectors, called $|1\rangle,|3\rangle$ and $|5\rangle$ respectively, giving us a 3 -dimensional Hilbert space $\mathcal{H}$. We also argue that picking options (2) or (4), represented by the vectors $|2\rangle$ and $|4\rangle$, does not necessarily means that the participant disagrees with (1) and (3) or (3) and (5) respectively. Keeping in mind the balanced property of Likert scales, places $|2\rangle$ symmetrically between $|1\rangle$ and $|3\rangle$ and $|4\rangle$ symmetrically between $|3\rangle$ and $|5\rangle$. Note that we can easily incorporate assumptions (e.g. balanced) from Likert scale theory into our model. This naturally leads to the structure depicted in figure 2 .

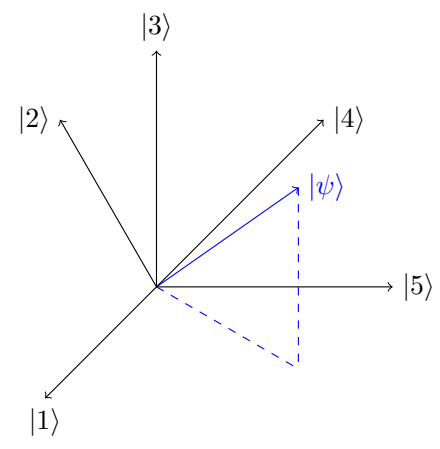

Fig. 2. The outcome '(i)' is represented by $|i\rangle$. The participant is represented by state vector $|\psi\rangle$.

Our implied structure in the outcomes does not impose restrictions on the agents. We can still model a person who doubts between (1) and (5) but not (3), by having a state vector equal to, for example, $|\psi\rangle=(1 / \sqrt{2}, 0,1 / \sqrt{2})$.

Our arguments about the (non)-excluding outcomes and resulting dimensions here are very superficial. One could, e.g., argue that option (3) should be symmetrical between (1) and (5), leading to a 2-dimensional Hilbert space. A meticulous investigation of Likert scales in this paradigm falls outside the scope of this paper. We only wish to show that it is possible to represent inherent ordinal structure in the outcomes, possibly combined with other theoretical assumptions or restrictions.

\section{Concluding remarks}

In this paper we propose some tentative first steps towards modeling ordinal scales using quantum-like techniques. After losing some of the restrictions used in the construction of projective measurements (and even lose restrictions placed upon POVMs), we use this lack of structure to impose new structure, now originating from the internal structure that ordinal outcomes exhibit. These techniques also allow for a reduction of the resulting dimensionality, as this can be- 
come problematic quickly in slightly more complex situations than the common examples seen in quantum cognition. We discuss the two biggest consequences of this approach, the first one being the total sum across all probabilities exceeding one and the second one being the loss of repeatability of outcomes. Exceeding one when adding the probabilities makes scaling necessary or requires the modeling of odds of outcomes instead of probabilities. We argue that the loss of repeatability is not as problematic as it seems at first and provide a possible interpretation of this phenomenon. Finally, we mention two possible applications. First, we give a short overview of an implementation already done in a game theoretic setting. Second, we propose the idea of applying our quantum-like ordinal system to model Likert scales.

This contribution is only a first step into modeling ordinal scales in a quantumlike way. The theoretical side of this story needs to be deepened, with a more thorough discussion of the concepts sketched out in section 3, next to investigating structures similar in role to Naimark's Theorem for POVMs ([8]). Also, more data-driven applications than the one presented here need to be formulated and statistically tested to investigate the true merit of this new approach.

\section{References}

1. Aerts, D., de Bianchi, M.S.: Beyond-quantum modeling of question order effects and response replicability in psychological measurements. CoRR abs/1508.03686 (2015), http://arxiv.org/abs/1508.03686

2. Aliakbarzadeh, M., Kitto, K.: Applying povm to model non-orthogonality in quantum cognition. In: Atmanspacher, H., Filk, T., Pothos, E. (eds.) Quantum Interaction, Lecture Notes in Computer Science, vol. 9535, pp. 284-293. Springer International Publishing (2016)

3. Blanco, M., Engelmann, D., Koch, A., Normann, H.T.: Preferences and beliefs in a sequential social dilemma. Games and Economic Behavior 87, 122-135 (2014)

4. Busemeyer, J., Bruza, P.: Quantum Models of Cognition and Decision. Cambridge University Press, Cambridge (2012)

5. Busemeyer, J., Pothos, E., Franco, R., Trueblood, J.: A quantum theoretical explanation for probability judgment errors. Physchol. Rev 118(2), 193-218 (2011)

6. Camparo, J.: A geometrical approach to the ordinal data of likert scaling and attitude measurements: The density matrix in psychology. Journal of Mathematical Psychology 57(1), 29-42 (2013)

7. Denolf, J., Martínez-Martínez, I., Josephy, H., Barque-Duran, A.: A quantum-like model for complementarity of preferences and beliefs in dilemma games. (submitted)

8. Gelfand, I., Neumark, M.: On the imbedding of normed rings into the ring of operators in hilbert space. Mat. Sbornik 54, 197-217 (1943)

9. Khrennikov, A., Basieva, I., Dzhafarov, E.N., Busemeyer, J.R.: Quantum models for psychological measurements: an unsolved problem. PloS one 9(10), e110909 (2014)

10. Martínez-Martínez, I., Denolf, J., Barque-Duran, A.: Do preferences and beliefs in dilemma games exhibit complementarity? In: Quantum Interaction, pp. 142-153. Springer (2015) 
11. Matvejchuk, M., Widdows, D.: Real-orthogonal projections as quantum pseudologic. In: Quantum Interaction, pp. 275-283. Springer (2015)

12. Moore, D.W.: Measuring new types of question-order effects: Additive and subtractive. The Public Opinion Quarterly 66(1), 80-91 (2002)

13. de Muynck, W.: Povms: A small but important step beyond standard quantum mechanics. Beyond the Quantum (eds T. Nieuwenhuizen, B. Mehmani, V. Špicka, M. Aghdami \& A. Khrennikov) pp. 69-79 (2007)

14. Nielsen, M.A., Chuang, I.L.: Quantum computation and quantum information. Cambridge university press (2010)

15. Pothos, E., Busemeyer, J.: A quantum probability explanation for violations of 'rational' decision theory. Proceedings of the Royal Society of London B: Biological Sciences pp. rspb-2009 (2009)

16. Spector, P.E.: Summated rating scale construction: An introduction. No. 82, Sage (1992)

17. Yearsley, J.M.: Advanced tools and concepts for quantum cognition: A tutorial. Journal of Mathematical Psychology. Special Issue on Quantum Probability (in press) (2016) 